\title{
Avaliação dos resultados entre a colecistectomia laparoscópica eletiva ou de urgência
}

\author{
Outcome assessment between elective or urgent laparoscopic cholecystectomy
}

\author{
Waleska Ramos Alvim Lescowicz ${ }^{1}$, Mylla Kaori Shimada Okuhara ${ }^{2}$, Rinaldo Danesi Pinto ${ }^{3}$
}

Lescowicz WRA, Okuhara MKS, Pinto RD. Avaliação dos resultados entre a colecistectomia laparoscópica eletiva ou de urgência / Outcome assessment between elective or urgent laparoscopic cholecystectomy. Rev Med (São Paulo). 2020 maio-jun.;99(3):266-71.

RESUMO: Introdução: Colecistectomia laparoscópica é tratamento padrão-ouro para colecistolitíase. É classificada em eletiva ou de urgência e existe uma quantidade pequena de publicações que comparam esses dois momentos de atendimento. Objetivo: Analisar os resultados de colecistectomias laparoscópicas eletivas ou de urgência quanto ao tempo entre internação e cirurgia, tempo de hospitalização, complicações, uso de antimicrobianos e de drenos, mortalidade e custo. Métodos: Análise retrospectiva de 368 pacientes submetidos à colescistectomia laparoscópica. Dados foram coletados do prontuário e da conta hospitalar dos pacientes. Resultados: Dos 368 pacientes, $277(75,3 \%)$ foram submetidos à colecistectomia laparoscópica eletiva e $91(24,7 \%)$ à de urgência. Dos submetidos à eletiva, $194(70,04 \%)$, eram mulheres. A idade média foi de $45,66 \pm 14,87$ anos. A permanência hospitalar média foi de $26,60 \pm 16,85$ horas. Utilizaram terapia antimicrobiana $2(0,72 \%)$ pacientes e dreno $16(5,78 \%)$ pacientes. Houve complicações em $6(2,17 \%)$ casos. O custo médio foi $3.157,76 \pm 1.882,22$ reais. Nas de urgência, $61(67,03 \%)$ eram mulheres. A idade média foi $51,36 \pm 19,08$ anos. A permanência hospitalar média

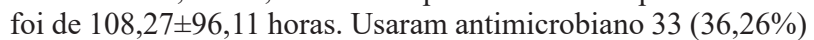
pacientes e dreno $21(23,08 \%)$ pacientes. Complicações ocorreram em $13(14,29 \%)$ pacientes. Dois $(2,20 \%)$ pacientes foram a óbito. O custo médio foi de $9.357,00 \pm 7.224,14$ reais. Analisando as variáveis, o custo médio das colecistectomias de urgência foi o triplo do das eletivas. Conclusão: Colecistectomias laparoscópicas de caráter urgente apresentam um custo consideravelmente mais elevado devido ao maior tempo entre internação e cirurgia, maior tempo de hospitalização, maior índice de complicações, mais uso de drenos e maior necessidade de terapia antimicrobiana, quando comparadas a procedimentos eletivos.

Descritores: Colecistectomia laparoscópica; Procedimentos cirúrgicos eletivos; Complicações intraoperatórias.

\begin{abstract}
Background: Laparoscopic cholecystectomy is gold standard treatment for cholecystolithiasis. It is classified as elective or urgent and there is a small amount of publications comparing these two moments of attendance. Aim: To analyze the results of elective and urgent laparoscopic cholecystectomy regarding the time between hospitalization and surgery, the length of hospitalization, complications, use of antimicrobials and drains, mortality, and cost. Methods: Retrospective analysis of 368 patients who underwent laparoscopic cholecystectomy. Data were collected from the medical records and from the hospital account of the patients. Results: Of 368 patients, 277 (75.3\%) underwent elective cholecystectomy and 91 (24.7\%) under emergency cholecystectomy. Of those submitted to the elective, $194(70.04 \%)$ were women. The average age was $45,66 \pm 14,87$ years. The average hospital stay was $26.60 \pm 16,85$ hours. Two $(0.72 \%)$ patients used antimicrobial therapy and $16(5.78 \%)$ patients used drain. There were complications in $6(2.17 \%)$ cases. The average cost was $3,157.76 \pm 1.882 .22$ reais. In emergency cholecystectomies, $61(67.03 \%)$ patients were women. The average age was $51.36 \pm 19,08$ years. The average hospital stay was $108.27 \pm 96,11$ hours. Antimicrobials were used by 33 (36.26\%) patients and drain by $21(23.08 \%)$ patients. Complications occurred in $13(14.29 \%)$ patients. Two $(2.20 \%)$ patients died. The average cost was 9,357.00 \pm 7.224 .14 reais. Analyzing the variables, the average cost of emergency cholecystectomies was three times the cost of electives. Conclusion: Urgent laparoscopic cholecystectomies have a considerably higher cost due to longer time between hospitalizations and surgeries, longer hospitalization time, higher complication rate, more use of drains and greater need for antimicrobial therapy when compared to elective procedures.
\end{abstract}

Headings: Cholecystectomy, laparoscopic; Elective surgical procedures; Intraoperative complications.

\footnotetext{
1. Acadêmica do Curso de Medicina da Universidade Regional de Blumenau (FURB), Departamento de Medicina, Blumenau, SC, Brasil. ORCID: https://orcid.org/0000-0002-0106-1097. Email: waleska.ral@hotmail.com.

2. Acadêmica do Curso de Medicina da Universidade Regional de Blumenau (FURB), Departamento de Medicina, Blumenau, SC, Brasil. ORCID: https://orcid.org/0000-0001-5203-6265.Email: myllaokuhara@hotmail.com.

3. Professor, Mestre da Universidade Regional de Blumenau (FURB) e Cirurgião no Hospital Santa Catarina, Serviço de cirurgia, Blumenau, SC, Brasil. ORCID: https://orcid.org/0000-0003-4847-6676. Email: pinto.rinaldo@gmail.com.

Endereço para correspondência: Waleska Ramos A. Lescowicz. Rua Ismael Carlos Correia, 22. Saguaçu, Joinville, SC. CEP: 89221-520.
} 


\section{INTRODUÇÃO}

Colecistectomia laparoscópica (CL) é considerada como o tratamento padrão-ouro para colecistolitíase. Ela substituiu a colecistectomia laparotômica como a primeira escolha de tratamento para cálculos biliares e colecistite ${ }^{1}$.

As colecistectomias podem ser classificadas em eletivas ou de urgência, sendo eletivas aquelas em que é possível escolher a data mais apropriada para a realização do procedimento, não havendo caráter de urgência ou emergência. Essas, em sua maioria, são realizadas após diversos exames que visam obter a melhor condição de saúde do paciente e reduzir ao máximo o risco de intercorrências. Já as de urgência são realizadas quando o paciente apresenta um quadro grave, como por exemplo uma colecistite aguda, que necessita de uma intervenção médica imediata ${ }^{2}$.

Um estudo realizado no Reino Unido ${ }^{3}$ comparou os dois tipos de cirurgia e mostrou que as cirurgias eletivas demandaram menor tempo médio operatório, menor incidência de complicações, como cardiovasculares e fístulas biliares e menos fatores de risco na conversão para laparotomia que as de urgência. $\mathrm{O}$ mesmo estudo mostrou que o tempo de internação para o paciente operado de urgência é praticamente o dobro.

Análise feita com os dados da colecistectomia na Michigan Surgical Quality Collaborative (USA) ${ }^{4}$, em todo o estado de Michigan (EUA), mostrou um tempo de permanência hospitalar significativamente aumentado em pacientes com colecistectomia de urgência. As taxas de morbidade e de mortalidade também foram maiores nas colecistectomias urgentes comparadas com as eletivas.

A colocação de dreno na colecistectomia laparoscópica tem a função de evitar coleções de bile e sangue, que futuramente podem requerer procedimentos abertos. Uma análise do Cochrane Database of Systematic Reviews, concluiu que não existem evidências que sustentem o uso de drenos em cirurgias eletivas. Por outro lado, observou-se que no manejo cirúrgico da colecistite aguda, o uso de dreno foi relatado pela maioria dos cirurgiões. A operação de urgência por colecistite aguda foi o principal motivo para a drenagem, seguido de derramamento de bile intra-operatório ${ }^{5}$.

Além disso, um fator de comparação importante é o custo dessas intervenções. O custo total da hospitalização é a soma de vários fatores, tais como aqueles relacionados com serviços de diagnóstico e terapia - incluindo exames complementares e procedimentos cirúrgicos - que são diferentes dependendo se a cirurgia foi eletiva ou de urgência. Isso pode causar variações nos custos para o mesmo procedimento cirúrgico ${ }^{6}$. Em 2016, na cidade de Fortaleza, Ceará (Brasil), foram alocados R \$ 2.682.666,88 com admissões por colelitíase e colecistite, representando $8,35 \%$ dos custos de hospitalização com doenças benignas do sistema digestório da cidade nesse período. Entre as internações por colelitíase, $73,68 \%$ foram para internação eletiva ${ }^{7}$.

"Eletivo ou de urgência, o fato é que existe quantidade pequena de publicações que comparam esses dois momentos de atendimento"s.

O objetivo deste estudo foi analisar comparativamente os resultados de colecistectomias eletivas e de urgência no contexto do tempo entre internação e cirurgia, tempo de hospitalização, complicações, uso de antimicrobianos, uso de dreno, mortalidade e custo global em um hospital privado.

\section{MÉTODOS}

Este estudo retrospectivo foi realizado através de consulta ao prontuário eletrônico e conta hospitalar de 368 pacientes submetidos à CL no Hospital Santa Catarina, da cidade de Blumenau - SC, Brasil, no período de novembro de 2016 a outubro de 2017.

Os pacientes foram separados entre os submetidos à $\mathrm{CL}$ eletiva e os submetidos à CL de urgência. Definiuse por colecistectomias eletivas aquelas em que foi possível escolher a data mais apropriada para a realização do procedimento, não havendo caráter de urgência ou emergência. E por colecistectomia de urgência as realizadas quando o paciente entrou no hospital pelo pronto socorro e apresentava um quadro agudo, como colecistite aguda ou cólica biliar. Foram excluídos os pacientes nos quais se realizou outro procedimento além da colecistectomia durante a cirurgia.

Foram então analisados nos prontuários eletrônicos gênero, faixa etária, tempo entre internação e cirurgia, tempo de hospitalização, uso de antimicrobianos, uso de dreno, complicações, desfecho (alta hospitalar ou óbito).

$\mathrm{Na}$ conta hospitalar foi analisado o custo global do procedimento (desde a entrada no hospital até a alta).

As CL foram realizadas uma equipe cirúrgica composta por 11 cirurgiões, os quais têm de 5 a 11 anos de experiência em cirurgias laparoscópicas.

Foi obtido consentimento escrito e informado de todos os pacientes incluídos no estudo antes da CL. O protocolo do estudo foi aprovado pelo comitê de ética local.

A análise dos dados e realização dos testes foi realizada através de software estatístico. Foram utilizados média e desvio padrão para descrição dos dados quantitativos e frequência para os dados qualitativos. Aplicou-se o teste de Kolmogorov-Smirnov para analisar quais variáveis apresentam distribuição paramétrica. As médias foram comparadas pelo teste " $t$ " de Student para as variáveis paramétricas ou teste U de Mann-Whitney para as variáveis não paramétricas. As frequências foram comparadas pelo teste do qui-quadrado. $O$ valor $p<0,05$ foi considerado estatisticamente significante. 


\section{RESULTADOS}

No grupo eletivo, a população foi de 277 (75,30\%) pacientes. Destes, 194 (70,04\%) foram do sexo feminino e $83(29,96 \%)$ do sexo masculino. A idade média foi de $45,66 \pm 14,87$ anos.

O tempo médio de permanência no hospital desse grupo foi de $26,60 \pm 16,85$ horas. E o tempo médio entre internação e cirurgia foi de $1,66 \pm 0,18$ horas.

Profilaxia antimicrobiana foi utilizada por $100 \%$ dos pacientes, seguindo protocolo hospitalar. Apenas $2(0,72 \%)$ pacientes fizeram uso de terapia antimicrobiana, no caso a associação de ciprofloxacina (200mg endovenoso de $8 \mathrm{em} 8$ hs) e metronidazol (500 mg de endovenosa a cada 8 horas). Dos profiláticos, a grande maioria fez uso de cefazolina $2 \mathrm{~g}$ intravenoso durante indução anestésica.

Uso de dreno ocorreu em 16 (5,78\%) pacientes e, desses, 14 usaram dreno tubular, um usou dreno de Kehr junto do tubular e um dreno suctor.

Foram identificados $6(2,17 \%)$ casos de procedimentos com intercorrências ou complicações, conforme a Tabela 1. Em trrês desses casos houve sangramento em leito hepático, sendo que um foi resolvido com rafia da lesão hepática e os outros dois necessitaram de um novo procedimento cirúrgico, tendo que realizar nova laparoscopia para rafia da lesão. Em um caso o paciente apresentou dor abdominal difusa e intensa, sem melhora com morfina e hipotensão (pressão arterial de $82 \times 60 \mathrm{mmHg}$ ), sendo internado na UTI. Um paciente teve crise hipertensiva na indução anestésica e outro apresentou dispneia a pequenos esforços, saturando 86\% em ar ambiente, sendo revertido após nebulização com broncodilatador. Nenhum paciente foi a óbito.

Tabela 1. Intercorrências ou complicações das CL eletivas

\begin{tabular}{l|c}
\hline Intercorrências ou Complicações & n \\
\hline Sangramento em leito hepático. & 3 \\
\hline $\begin{array}{l}\text { Dor abdominal difusa e intensa, sem melhora } \\
\text { com morfina e hipotensão (pressão arterial de } \\
82 x 60 \mathrm{mmHg} \text { ), sendo internado na UTI. }\end{array}$ & 1 \\
\hline Crise hipertensiva na indução anestésica & 1 \\
\hline Dor, distensão abdominal e febre. & 1 \\
\hline Hipertensão no intraoperatório. & 1 \\
\hline $\begin{array}{l}\text { Dispneia a pequenos esforços, saturando 86\% em } \\
\text { ar ambiente, sendo revertido após nebulização com } \\
\text { broncodilatador. }\end{array}$ & 1 \\
\hline
\end{tabular}

O custo médio do procedimento foi de $3.157,76 \pm 1.882,22$ reais.

Já no grupo dos pacientes operados em regime de urgência, a população foi de 91 (24,70\%) pacientes. Destes, $61(67,03 \%)$ eram do sexo feminino e $30(32,97 \%)$ do sexo masculino. A média da idade foi 51,36 $\pm 19,08$ anos.

A permanência hospitalar média foi de 108,27 $\pm 96,11$ horas. E a média do tempo entre internação e cirurgia foi de 44,29 $\pm 35,35$ horas.

Assim como nos eletivos, $100 \%$ dos pacientes utilizaram profilaxia antimicrobiana com cefazolina $2 \mathrm{~g}$ intravenoso durante indução anestésica. Uso terapêutico ocorreu em $33(36,26 \%)$ pacientes, sendo novamente a combinação de ciprofloxacina (200mg endovenoso de $8 \mathrm{em}$ 8) e metronidazol ( $500 \mathrm{mg}$ de endovenosa a cada 8 horas) a mais utilizada para esse fim.

Uso de dreno ocorreu em 21 (23,08\%) pacientes e, desses, 19 usaram dreno tubular, 1 usou dreno de Blake e 1 dreno Penrose $n^{\circ} 1$.

Intercorrências ou complicações ocorreram em 13 (14,29\%) pacientes, 2 destes tiveram retenção urinária, os outros, cada um apresentou uma complicação diferente, sendo elas especificadas na Tabela 2. Dois $(2,20 \%)$ pacientes foram a óbito.

Tabela 2. Intercorrências ou complicações das CL de urgência

\begin{tabular}{l|c}
\hline Intercorrências ou Complicações & n \\
\hline Retenção urinária. & 2 \\
\hline Broncosespasmo pós extubação. & 1 \\
\hline $\begin{array}{l}\text { Instabilidade hemodinâmica, insuficiência renal } \\
\text { aguda, cianose central e necrose extremidades. }\end{array}$ & 1 \\
\hline Dor, distensão abdominal e febre. & 1 \\
\hline Hipertensão no intra-operatório. & 1 \\
\hline $\begin{array}{l}\text { Rebaixamento do nível de consciência no pós- } \\
\text { operatório, derrame pleural sendo realizada } \\
\text { toracocentese. Derrame pericárdico e parada } \\
\text { cardiorrespiratória. }\end{array}$ & 1 \\
\hline $\begin{array}{l}\text { Queda de saturação na sala cirúrgica (75\% em ar } \\
\text { ambiente) com cianose de extremidades, tendo } \\
\text { que adiar a cirurgia. Após CL fez evento séptico, } \\
\text { insuficiência renal aguda, anemia e anasarca. }\end{array}$ & 1 \\
\hline $\begin{array}{l}\text { Leucocitose, função renal alterada, derrame pleural, } \\
\text { anemia, insuficiência respiratória aguda após } \\
\text { transfusão de hemácias e bacteremia. }\end{array}$ & 1 \\
\hline $\begin{array}{l}\text { Pico hipertensivo. } \\
\text { Tromboembolismo pulmonar. }\end{array}$ & 1 \\
\hline $\begin{array}{l}\text { Instalada sonda nasogástrica longa durante o } \\
\text { procedimento. }\end{array}$ & 1 \\
\hline $\begin{array}{l}\text { Fístula biliar, rebaixamento do nível de consciência, } \\
\text { broncoespasmo com necessidade de intubação, choque } \\
\text { séptico e infecção por e. coli em líquido abdominal. }\end{array}$ & 1 \\
\hline
\end{tabular}

O custo médio do procedimento foi de $9.357,00 \pm 7.224,14$ reais.

Analisando comparativamente os resultados, em relação à idade $(p=0,02444)$, tempo entre internação 
e cirurgia $(p<0.00001)$ e tempo total intra-hospitalar $(\mathrm{p}<0.00001)$, observou-se diferença com significância estatística nas comparações entre CL eletiva e de urgência. Assim, tem-se que as cirurgias de urgência tiveram pacientes mais idosos, maior espera desde a internação até a cirurgia e maior duração da hospitalização. Nota-se através da Tabela 3 que a média destas variáveis nas cirurgias de urgência foram significativamente maiores.
Observa-se também que o custo médio das CL de urgência foi praticamente o triplo das CL eletivas $(\mathrm{p}<0.00001)$.

Analisando as variáveis qualitativas, a ocorrência de intercorrências ou complicações $(p<0.00001)$, uso de dreno $(p<0.00001)$ e uso de terapia antimicrobiana $(p<0.00001)$, foram mais recorrentes nas CL de urgência em comparação às eletivas (Tabela 4).

Tabela 3. Análise das variáveis quantitativas em relação a CL de urgência ou eletivas

\begin{tabular}{lccccc}
\hline \multirow{2}{*}{ Variável } & \multicolumn{2}{c}{ Eletivas $(\mathbf{n = 2 7 7 )}$} & \multicolumn{2}{c}{ Urgência (n=91) } & p \\
\cline { 2 - 5 } & Média & DP & Média & DP & \\
\hline Idade (anos) & 45,66 & 14,87 & 51,36 & 19,08 & 0,02444 \\
Tempo entre internação e cirurgia (horas) & 1,66 & 0,18 & 44,29 & 35,35 & $<0.00001$ \\
Tempo total no hospital (horas) & 26,60 & 16,85 & 108,27 & 96,11 & $<0.00001$ \\
Custo (reais) & $3.157,76$ & $1.882,22$ & $9.357,00$ & $7.224,14$ & $<0.00001$ \\
\hline
\end{tabular}

Tabela 4. Análise das variáveis qualitativas em relação a CL de urgência ou eletivas

\begin{tabular}{lccccc}
\hline \multirow{2}{*}{ Variável } & \multicolumn{2}{c}{ Eletivas } & \multicolumn{2}{c}{ Urgência } & p \\
\cline { 2 - 5 } & $\mathbf{n}$ & $\mathbf{\%}$ & $\mathbf{n}$ & $\mathbf{\%}$ & $<0.00001$ \\
\hline Complicações & 6 & 2,70 & 13 & 14,29 & $<0.00001$ \\
Uso de Antimicrobianos & 2 & 1,25 & 33 & 51,56 & $<0.00001$ \\
Uso de Dreno & 16 & 5,78 & 21 & 23,08 & $\mathrm{a}$ \\
Óbito & 0 & 0 & 2 & 2,20 & \\
\hline
\end{tabular}

a. Nenhuma estatística foi calculada.

\section{DISCUSSÃO}

Este trabalho analisou os resultados das colecistectomias eletivas e de urgência buscando comparar suas diferenças quanto ao tempo entre a internação e a cirurgia, o tempo de hospitalização, as complicações, o uso de antimicrobianos, o uso de dreno, os desfechos e o custo global do procedimento. As variáveis incluídas foram analisadas objetivando investigar sua relação com os procedimentos cirúrgicos, eletivos ou de urgência.

Ao analisar o sexo dos pacientes foi encontrada uma prevalência de $70,04 \%$ do sexo feminino e de $29,96 \%$ de pacientes do sexo masculino nas cirurgias eletivas. Em relação às cirurgias de urgência, a prevalência foi de $67,03 \%$ para mulheres e de $32,97 \%$ para homens. Uma maior prevalência de mulheres é compatível com o encontrado na literatura médica.

Em relação à idade, uma média de 45,66 e 51,36 anos foi encontrada para as cirurgias eletivas e de urgência, respectivamente. Observa-se uma média de idade maior em cirurgias de caráter urgente pelo fato da incidência de colecistite aguda, e de outras agudizações ser significantemente mais alta em pacientes com idade mais avançada do que em pacientes jovens. Além disso, pacientes idosos são mais propensos a ter um histórico prolongado de doenças vesiculares e sofrerem agudizações ${ }^{9}$.

A média do tempo de internação até a cirurgia foi maior nas cirurgias de urgência, 44,29 horas, em consequência do tempo necessário para confirmação do diagnóstico e estabilização do paciente para o procedimento cirúrgico. Nas cirurgias eletivas, pelo fato de os pacientes já terem passado por consultas prévias, realizado diversos exames e possuírem o diagnóstico, a média foi de 1,66 horas.

O tempo total de internação também foi maior para as cirurgias de urgência, decorrente do maior tempo de internação até a cirurgia e do pós-operatório. Esses pacientes ficaram internados em média 108,26 horas, enquanto os pacientes eletivos ficaram em média 26,6 horas. A colecistite aguda, e outras agudizações têm relação 
com uma permanência maior no hospital após uma CL por apresentarem maiores taxas de conversão, uso de dreno após a cirurgia e complicações durante e após a cirurgia ${ }^{10}$.

Considerando intercorrências os eventos aleatórios, não desejáveis, mas possíveis de acontecer, que têm resolução breve e com risco baixo de comprometer a recuperação pós-operatória e complicações os eventos de causa definida, que têm uma resolução alongada e colocam em risco a evolução pós-operatória, observouse que não somente a porcentagem como também a gravidade dos eventos da cirurgia eletiva foram menores. Entre os pacientes eletivos, 2,17\% tiveram algum tipo de intercorrência, destes, apenas $1(0,36 \%)$ foi uma complicação, o paciente que apresentou hipotensão (pressão arterial de $82 \times 60 \mathrm{mmHg}$ ) e foi internado na UTI, os outros eventos foram todos intercorrências. Já nos pacientes de urgência, que contabilizaram 14,29\% intercorrências ou complicações, 4 (4,40\%) tiveram complicações muito graves, como tromboembolismo pulmonar, choque séptico, parada cardiorrespiratória e instabilidade hemodinâmica com insuficiência renal aguda.

As complicações ocorrem com mais frequência nos pacientes de urgência, pois esses, em sua maioria, apresentam quadro de colecistite aguda e possuem valores aumentados de marcadores inflamatórios. Além disso, se a ultrassonografia do paciente apresentar um empiema ou aumento da espessura da parede vesicular acima de três milímetros, há um risco ainda maior de complicações ${ }^{11}$.

Todos os pacientes eletivos fizeram uso de profilaxia antimicrobiana, sendo que na maioria foi administrado cefalozina. Em dois pacientes $(0,72 \%)$ foi necessária terapia antimicrobiana, associando-se ciprofloxacina com metronidazol. Uma pesquisa realizada por Uludag et al. ${ }^{12}$ comparou um grupo para o qual foi administrado profilaxia antimicrobiana a outro para o qual administrou-se um placebo. Ambos os grupos eram constituídos por pacientes que seriam submetidos a cirurgias eletivas. Concluiu-se que a profilaxia antimicrobiana não influenciou na incidência de infecções do sítio cirúrgico e, por esse motivo, não é necessária em colecistectomias laparoscópicas eletivas em pacientes de baixo risco. Souza et al. ${ }^{13}$ chegou à mesma conclusão em seu estudo no qual foram analisados 199 pacientes eletivos. Todavia, segundo Uchiyama et al. ${ }^{14}$, as complicações associadas a infecções pósoperatórias mostraram-se reduzidas com o uso de profilaxia antimicrobiana administrada a uma hora da colecistectomia laparoscópica.

Assim como os pacientes que realizaram cirurgia eletiva, em todos os pacientes urgentes foi administrado profilaxia antimicrobiana. Terapia antimicrobiana foi utilizada em 33 pacientes (36,26\%), sendo a associação de ciprofloxacino com metronidazol a mais utilizada. $\mathrm{O}$ eletivos.

Participação dos Autores: Lescowicz WRA: Revisão de literatura, coleta de dados, análise de dados, redação do artigo, elaboração de tabelas e revisão final. Okuhara MKS: Revisão de literatura, coleta de dados, análise de dados, redação do artigo, elaboração de tabelas e revisão final.
Guideline de Tokio $^{15}$ publicado em 2018 recomenda a administração de antimicrobianos até 24 horas depois de realizada a cirurgia em pacientes com colecistite aguda leve e durante quatro a sete dias em casos moderados ou graves, uma vez que controlado o foco da infecção. A redução do uso incorreto de antimicrobianos é de grande importância no sentido de diminuir as complicações causadas por esses medicamentos (como alergias e sintomas gastrointestinais), os custos e a resistência bacteriana ${ }^{16,17}$.

Quanto ao uso de dreno, foi necessária sua utilização em $5,78 \%$ dos pacientes eletivos, isto é, em 16 pacientes de um total de 277. Em sua maioria, o dreno escolhido foi o tubular, colocado em 14 indivíduos. Observa-se que em pacientes que foram submetidos a cirurgias de urgência o uso de drenos é mais frequente, $23,08 \%$, o equivalente a 21 pacientes dos 91 analisados. Destes, 19 utilizaram dreno tubular, e os outros dois, os drenos de Blake e Penrose $n^{\circ} 1$.

A alta hospitalar foi o desfecho para todos os pacientes eletivos e, para $97,8 \%$ dos pacientes submetidos à colecistectomia de urgência. A taxa de mortalidade encontrada nesse estudo, de $2,20 \%$ dos pacientes Bardram $^{18}$. Outro ponto importante a ser considerado é idade desses pacientes que foram a óbito, média de 88,5 anos. A idade avançada, as comorbidades associadas comuns nesse grupo de pacientes e o caráter de urgência da cirurgia em consequência de um quadro agudo são fatores que aumentam o risco cirúrgico, de complicações e, consequentemente, a taxa de mortalidade ${ }^{9,19,20}$. Entretanto, temos que considerar que a taxa de mortalidade total, considerando tanto os pacientes eletivos quando os de diz que a taxa de mortalidade das colecistectomias é de $0.5 \%$ nos pacientes com mais de 50 anos $^{2}$.

O custo médio das cirurgias de caráter urgente foi aproximadamente três vezes maior do que os das cirurgias eletivas, em consequência de fatores como o tempo prolongado entre a internação e a cirurgia, o tempo total de permanência hospitalar, a necessidade do manejo de complicações, utilização de drenos e terapia antimicrobiana. Tratar precocemente pacientes que ainda não apresentam um quadro inflamatório agudo, por meio de cirurgia eletiva, minimiza o custo total do tratamento ${ }^{21,22}$.

\section{CONCLUSÃo}

Colecistectomias laparoscópicas de caráter urgente apresentam um custo consideravelmente mais elevado devido ao maior tempo entre internação e cirurgia, maior tempo de hospitalização, maior índice de complicações, mais uso de drenos e maior necessidade de terapia antimicrobiana, quando comparadas a procedimentos de urgência, é semelhante à publicada por Harboe e urgência, foi de $0,54 \%$, condizendo com a literatura, que 
Pinto RD: Orientação do projeto e revisão final.

\section{REFERÊNCIAS}

1. Duman K, Sezer K.H, Yılmaz F, Akın M.L. The clinical outcome of traditional laparoscopic cholecystectomy. Gaziantep Med J. 2013;19(1):35-9. doi: 10.5455/GMJ-302012-118.

2. Conde LM, Tavares PM, Quintes JLD, Chermont RQ, Perez CA. Laparoscopic management of cholecystocolic fistula. Abcd, Arq Bras Cir Dig. 2014;27(4):285-7. doi: 10.1590/ S0102-67202014000400013.

3. Kanakala V, Borowski DW, Pellen MGC, Dronamraju SS, Woodcock SAA., Seymour K, et al. Risk factors in laparoscopic cholecystectomy: a multivariate analysis. Int J Surg. 2011;9(4):318-23. doi: 10.1016/j.ijsu.2011.02.003.

4. To KB, Cherry-Bukowiec JR, Englesbe MJ, Terjimanian MN, Shijie C, Campbell DA, et al. Emergent versus elective cholecystectomy: conversion rates and outcomes. Surg Infect. 2013;14(6):512-9. doi: 10.1089/sur.2012.160.

5. Lucarelli P, Picchio M, Martellucci J, Angelis F, Filippo A, Stipa F, et al. Drain after laparoscopic cholecystectomy for acute calculous cholecystitis. A pilot randomized study. Indian J Surg. 2015;77(4):288-92. doi: 10.1007/s12262-012-0797-9.

6. Brasil. Ministério da Saúde. Portaria nº 1919. Redefine, no âmbito do Sistema Único de Saúde - SUS, a prestação de procedimentos cirúrgicos eletivos. Diário Oficial da União, Brasília. 2010;197(7):479-521.

7. Datasus. Informações de saúde. Disponível em http://datasus. gov.br. Acesso em: 03/05/2018.

8. Felício SJO, Matos EP, Cerqueira AM, Farias KWS, Silva RA, Torres MO. Mortalidade da colecistectomia videolaparoscópica de urgência versus operação eletiva para colecistite aguda. ABCD Arq Bras Cir Dig. 2017;30(1):47-50. doi: 10.1590/0102-67202017000100013.

9. Polychronidis A, Botaitis S, Tsaroucha A, Tripsianis G, Bounovas A, Pitiakoudis M, Simopoulos C. Laparoscopic cholecystectomy in elderly patients. J Gastrointestin Liver Dis. 2008;6:273-84. Available from: http://www.jgld. ro/2008/3/10.pdf.

10. Wasana K, Trichak S, Sahattaya P, et al. Predictive factors for a long hospital stay in patients undergoing laparoscopic cholecystectomy. Int J Hepatol. 2017;5:145-57. doi: $10.1155 / 2017 / 5497936$.

11. Radunovic M, Lazovic R, Popovic N, et al. Complications of laparoscopic cholecystectomy: our experience from a retrospective analysis. J Med Sci. 2016;4(4):641-6. doi: 10.3889/oamjms.2016.128.
12. Uludag M, Yetkin G, Citgez B. The role of prophylactic antibiotics in elective laparoscopic cholecystectomy. JSLS. 2009;13(3):337-41. Available from: https://www.ncbi.nlm. nih.gov/pmc/articles/PMC3015970/.

13. Souza HP, Breigeiron R, Cunha HM, Deves E. Antibioticoprofilaxia na colecistectomia videolaparoscópica eletiva: estudo prospectivo randomizado e duplo cego. Rev Col Bras Cir. 2008;35(3):168-72. doi: 10.1590/S010069912008000300006 .

14. Uchiyama K, Kawai M, Onishi H, et al. Preoperative antimicrobial administration for prevention of postoperative infection in patients with laparoscopic cholecystectomy. Dig Dis Sci. 2003;48(10):1955-9. doi: 10.1023/a:1026114203622.

15. Gomi H, Solomkin, JS, Schlossberg, D et al. Tokyo Guidelines 2018: antimicrobial therapy for acute cholangitis and cholecystitis. J Hepatobiliary Pancreat Sci. 2018;25:3-16. doi: $10.1002 /$ jhbp.518.

16. Pellegrini P, Campana JP, Dietrich A, et al. Protocol for extended antibiotic therapy after laparoscopic cholecystectomy for acute calculous cholecystitis (Cholecystectomy Antibiotic Randomised Trial, CHART). BMJ Open. 2015;5:109-15. doi: 10.1016/j.surg.2018

17. Regimbeau JM, Fuks D, Pautrat K, et al. Effect of postoperative antibiotic administration on postoperative infection following cholecystectomy for acute calculous cholecystitis: a randomized clinical trial. JAMA. 2014;312:145-54. doi: 10.1001/jama.2014.7586.

18. Harboe K, Bardram L. Nationwide quality improvement of cholecystectomy: results from a national database. Int J Qual Health Care. 2011;23(5):565-73. doi: 10.1093/intqhe/mzr041.

19. Ansaloni L, Pisano M, CoccoliniF, Peitzmann AB, Fingerhut A, Catena F, Agresta F, et al. 2016 WSES guidelines on acute calculous cholecystitis. World J Emerg Surg. 2016;11(1):25. doi: 10.1186/s13017-016-0082-5.

20. Sandblom G, Videhult P, Crona GY, Svenner A, Sadr-Azodi O. Mortality after a cholecystectomy: a population-based study. HPB (Oxford). 2014;17(3):239-43. doi: 10.1111/hpb.12356.

21. Carvalho RWF, Pereira CU, Laureano Filho JR, Vasconcelos BCE. O paciente cirúrgico parte II. Rev Cir Traumatol Bucomaxilofasc. 2011;11(4):9-12.

22. Schwartz DA, Shah AA, Zogg CK et al. Operative delay to laparoscopic cholecystectomy: Racking up the cost of health care. J Trauma Acute Care Surg. 2015;79:15-21. doi: 10.1097/ TA.0000000000000699.

Recebido: 21/06/2019

Aceito: 14/05/2020 